\title{
A Technical Approach to the Energy Blockchain in Microgrids
}

\author{
M.L. Di Silvestre, P. Gallo, M. G. Ippolito, E. Riva Sanseverino, and G. Zizzo, Senior Member, IEEE
}

\begin{abstract}
The present paper considers some technical issues related to the "energy blockchain" paradigm applied to microgrids. In particular, what appears from the study is that the superposition of energy transactions in a microgrid creates a variation of the power losses in all the branches of the microgrid. Traditional power losses allocation in distribution systems takes into account only generators while, in this work, a real-time attribution of power losses to each transaction involving one generator and one load node is done by defining some suitable indices. Besides, the presence of P-V nodes increases the level of reactive flows and provides a more complex technical perspective. For this reason, reactive power generation for voltage support at $P-V$ nodes poses a further problem of reactive power flow exchange, that is worth of investigation in future works in order to define a possible way of remuneration. The experimental section of the paper considers a Medium Voltage microgrid and two different operational scenarios.
\end{abstract}

Index Terms-Transactive energy; Blockchain; Energy blockchain; Microgrids; Power losses; Reactive Power; P2P.

\section{INTRODUCTION}

$\mathrm{T}$ HE energy blockchain is a distributed protocol devoted to energy transactions between generation and load nodes in power systems. "Transactive energy" appears as one of the most interesting technologies in the 2017 Gartner Hype-cycle [1]. As a matter of fact, several startups are flourishing around the Bitcoin technology for crypto-currency [2], showing the interest around the possibility of managing certified transactions on the Internet, without the need of intermediaries and third-party entities.

Countries, such as the US, where distributed generation (DG), microgrids and energy trading between neighbors are largely present, host probably the most suitable infrastructures and regulatory framework for this kind of technology. Indeed, among the most recent projects, the microgrid in President street (NY City) is based on the blockchain technology for managing energy transactions [3].

The significant time reduction for the management of economic transactions and the possibility to get rid of third party authorities are disruptive elements for the energy sector. Any need for an intermediary between two parties is removed:

M. L. Di Silvestre, P. Gallo, M. G. Ippolito, E. Riva Sanseverino and G Zizzo are with Department of Energy, Information Engineering and Mathematical Models (DEIM), University of Palermo, Palermo, I-90128, Italy. switching to a decentralized energy system, and detaching the related financial transactions from a centralized control unit, can be considered as another step towards a full decentralization. As an example, in [4] the authors define a localized Peer-to-Peer (P2P) energy trading model for buying and selling electricity among Plug-in Hybrid Electric Vehicles (PHEV). The model shows the potential of blockchain for improving transactions security.

For these reasons, electrical utilities are more and more interested in energy blockchain applications, starting cooperation initiatives and joint ventures.

The energy exchange is different from any other exchange of goods. Indeed, strong technical constraints reduce the realistic possibility of free energy exchanges between generators and loads. In the case of a microgrid, the decentralized generation of large amounts of energy, to be exchanged between nearby nodes, may produce unwanted reactive power circulation, while reducing active power production capability. Moreover, it is not possible to devise a precise physical transaction costs attribution.

Another issue to consider for a correct costs allocation is the identification of the type of microgrid ownership. A recent report from GTM-Research [5] classifies microgrid ownership into three classes: end user owned, third party owned, mixed ownership (microgrids with presence of different ownerships, in most case including public institutions, utilities and private end-users). In the second and third cases, the precise losses allocation for each energy transaction is a crucial issue, given that transactions happen among different entities.

In recent years, some applications of the blockchain technology for energy transactions in microgrids have appeared [6]-[7], however the concurrent use of the blockchain for the technical operation of the distribution network is quite new [8].

This paper goes beyond the economic aspects of energy transactions and uses the blockchain to make distributed decisions for technical operations on the grid.

In this context, the paper provides a threefold contribution for microgrid: (i) reviews different blockchain components and presents specific requirements for microgrids (Table I); (ii) proposes the blockchain for handling energy losses allocation; (iii) defines new timing for transacting both intended energy exchanges and their losses.

After a preliminary analysis of the problem, this paper

(e-mail: marialuisa.disilvestre@unipa.it; pierluigi.gallo@unipa.it; marianogiuseppe.ippolito@unipa.it; $\quad$ eleonora.rivasanseverino@unipa.it; gaetano.zizzo@unipa.it) 
provides some technical hints for using the blockchain technology for transactive energy. Furthermore, some exemplary relevant scenarios are studied for showing the effects of concurrent energy flows and their associated losses. In order to compare the proposed scenarios, the same transactions are considered in different grid configurations.

Energy transactions begin at different times and have different duration, therefore the relevant power flows may overlap. The tracing of the power flows [9] provides evidence about the following issues: each incoming transaction modifies the voltage profile and, consequently, the distribution of the branch currents and the associated energy losses; the flows of reactive and active power significantly change during each transaction; the attribution of energy losses to energy transactions is a complex task.

As far as the latter issue is concerned, this paper suggests some indices for attributing energy losses to physical transactions in microgrids. Of course, once the physical effect of a given transaction is technically assessed, it will be easier for generators to activate smart contracts between them and create an associated remuneration system. The scientific literature on the topic presents various methods for attributing losses to a set of distributed generators in a microgrid [10], [11]. Nevertheless, these methods do not take into account the issue of attributing the energy losses to each specific transaction taking place at distribution level between a couple generatorload.

Finally, the experiments presented in this paper show that the voltage support function and the availability of reactive power in microgrids become critical, since large reactive power flows may appear for voltage support, reducing the production potential of each generator.

\section{THE BLOCKCHAIN TECHNOLOGY}

Blockchain was originally used for tracking economic transactions without the need of a trusted intermediary institution (e.g. a bank). Even if born for economic transactions, in applications like Bitcoin, the blockchain demonstrates to be a disruptive and foundational technology that can be applied also to other fields, in all scenarios where distributed approaches are preferable over centralized ones. The use of blockchain spans from smart contracts [12] to personal data protection [13], including genomics, health, academic publishing, product traceability [14], voting and predictions [15].

The blockchain technology uses the public-key cryptography for eliminating the double-spending problem, which is one of the basic issues for crypto currencies. The double-spending problem appears when a digital token corresponding to a 'digital coin' can illegitimately be spent more than once. To prevent double-spending, actors use their private key to sign digitally a transfer of tokens from one actor to another and a database of spent tokens is kept. Real physical resources are required to mine new coins (e.g. energy spent for computation), which are verified through proofs of work. In the blockchain, actors are represented only by their keys, therefore their realworld identities are not disclosed.
The blockchain is a distributed ledger that contains a continuously growing list of data records called blocks [16]. Blocks can contain different types of transactions (financial, energy, goods, etc.), data or even logic and programs. Blocks are time-stamped, unalterable, verifiable, and connected each other in a chronological (chained) sequence. Users maintain a copy of the blockchain and any modification of blocks content or order is immediately revealed by a fast check on block validity

In case of energy purchase or selling, blocks can be organized in tables containing details as: generator ID (source), load ID (destination), amount of transferred energy, timestamp, duration and power profile. Additionally, this paper includes also presumed associated energy losses inside transactions.

Blocks include the output of a hash algorithm (e.g. Secure Hash Algorithm SHA256) computed on the previous block [17]. Hash algorithms receive variable-length messages and produce fixed-length digests that cannot be traced back to the original messages. Hash functions are collision resistant, i.e. it is extremely difficult to find two different messages having the same digest. The hash function receives as input the blockID, the actual transaction(s), a copy of the hash of the previous block and a nonce (number once). The nonce is a number specifically mined so that the resulting hash verifies specific conditions that occur on valid blocks, for example, the hash begins with a given number of zeros, as provided by the Hashcash mechanism [18]. Blocks integrity can be checked by computing one hash function and, if one block is changed, the condition on the hash is no more verified. A new valid nonce can be mined to make a modified block appears as valid. However, even in such a case, the lack of integrity is revealed because the block does not match the hash in the blockchain owned by the majority of users.

The chaining mechanism provides an extra protection: even the smallest modification on a block invalidates that block and all the following ones. This would require to mine new nonces for the modified block and all its successors, which becomes computationally heavier the older the modified block is. Furthermore, the validity of the whole chain can be checked by verifying only the hash of the last block.

The blockchain is a powerful and flexible technology and it can be customized in several different ways to operate at an optimal level depending on the specific application. In fact, the blockchain is not a one-size-fits-all solution, therefore smart grid experimenters look for the most suitable solution in the blockchain technology for handling energy transactions and technical operations and permitting smart contracts.

Table I individuates the following features that are relevant for choosing the most suitable microgrid-oriented blockchains:

- Consensus mechanism: the choice of the consensus mechanism affects energy consumptions in microgrids. Various possible solutions are listed in Table I, like Proof of Work (PoW), Proof of Stake (PoS), Proof of Activity $(\mathrm{PoA})$, Proof of Burn (PoB) and Proof of Elapsed Time (PoET);

Speed: the speed can affect the application in energy context, since the contracting should take place in quasi- 
real time to be more effective on balancing issues;

Permission model: defines the nodes enabled to read/write transactions. This can be permissionless (public), permissioned (restricted to a number of nodes) or private. In the energy field, it is most likely that permissioned blockchains will apply;

- Resilience: for blockchains is the capability to resist to attacks and malicious behaviors. This is analogous to the "fault ride through" capability of distributed generators in microgrids, providing an extra level of reliability.

In Table $\mathrm{I}, \mathrm{n}$ is the total number of nodes of the microgrid and $\mathrm{f}$ is the maximum number of faulty nodes that the system can face.

In accordance to Kerckhoff's principle, which states that secure cryptosystems should be publicly known about all aspects except the key, Table I includes only open source blockchains, with special focus on those for microgrids. This analysis includes elements reported in [19], with focus on aspects mainly dependent on the consensus algorithm and the used proving mechanism. PoW and mining require significant energy for computation, therefore they are not sustainable for green applications. In fact, during 2014 the energy for Bitcoin mining was comparable to Ireland's electricity consumption [20]. The use of the blockchain technology in microgrids must not be energy consuming, therefore, $\mathrm{PoS}$ is the preferred option, where decisions are taken by nodes who held stakes in the system (money, storage, power capacity, etc.).

The blockchain initially established itself as a totally decentralized, permissionless and fully public technology. However, today three different categories of blockchain are

TABLE I. MOST RELEVANT DISTRIBUTED LEDGERS USABLE FOR ENERGY TRANSACTIONS IN MICROGRIDS.

\begin{tabular}{|c|c|c|c|c|c|c|}
\hline $\begin{array}{c}\text { Name } \\
\text {--- } \\
\text { Github link }\end{array}$ & $\begin{array}{l}\text { Description and } \\
\text { consensus }\end{array}$ & $\begin{array}{c}\text { Speed } \\
\text { [tps] }\end{array}$ & Permissioned & $\begin{array}{c}\text { Resilience } \\
\text { against faults } \\
\text { / adversarial } \\
\text { nodes }\end{array}$ & $\begin{array}{l}\text { Development } \\
\text { notes }\end{array}$ & Note \\
\hline $\begin{array}{c}\text { Ethereum } \\
\text {--- } \\
\text { https://github.com/eth } \\
\text { ereum }\end{array}$ & $\begin{array}{l}\text { Proof-of-work (PoW) with } \\
\text { mining. } \\
\text { Shifting to Proof of Stake } \\
\text { (PoS) }\end{array}$ & $\sim 100$ & NO & $\mathrm{n} / \mathrm{a}$ & $\begin{array}{l}\text { Mainly Go or } \\
\text { C++ } \\
\text { LGPL3.0 } \\
\text { High } \\
\text { development } \\
\text { activity } \\
\end{array}$ & $\begin{array}{l}\text { Pluggable components. Rewards for } \\
\text { mined blocks. }\end{array}$ \\
\hline $\begin{array}{c}\text { Tendermint } \\
--- \\
\text { https://github.com/ten } \\
\text { dermint/tendermint }\end{array}$ & $\begin{array}{c}\text { Variant of PBFT } \\
\text { PoS } \\
\text { Validators "bond" their } \\
\text { currency holdings that are } \\
\text { lost if they misbehave }\end{array}$ & Thousands & YES & $\begin{array}{c}\mathrm{f}<\mathrm{n} / 3 \\
\text { security still } \\
\text { under } \\
\text { validation }\end{array}$ & $\begin{array}{l}94.1 \% \text { Go } \\
\text { still in alpha } \\
\text { Apache License } \\
\text { Version } 2.0\end{array}$ & $\begin{array}{l}\text { Apps for Application BlockChain } \\
\text { Interface (ABCI) } \\
\text { Include traceability, voting, invoicing } \\
\text { and High Frequency Energy Trading. } \\
\text { Uses validators. }\end{array}$ \\
\hline $\begin{array}{c}\text { HydraChain } \\
--- \\
\text { https://github.com/hy } \\
\text { drachain/ }\end{array}$ & $\begin{array}{l}\text { Hashgraph consensus } \\
\text { protocol only in tech } \\
\text { report. } \\
\text { Based on Babble }\end{array}$ & 250000 & YES & $\mathrm{n} / \mathrm{a}$ & $\begin{array}{l}\text { 98.6\% Python } \\
\text { Low } \\
\text { development } \\
\text { activity } \\
\text { MIT License. }\end{array}$ & $\begin{array}{c}\text { Ethereum extension, } \\
\text { inspired by Tendermint. } \\
\text { Asynchronous, no leaders, no round } \\
\text { robin, no PoW } \\
\text { Patented Hashgraph }\end{array}$ \\
\hline $\begin{array}{c}\text { Sawtooth Lake } \\
--- \\
\text { https://github.com/hy } \\
\text { perledger/sawtooth- } \\
\text { core }\end{array}$ & $\begin{array}{l}\text { General-purpose smart } \\
\text { contracts } \\
\text { Proof of Elapsed Time } \\
\text { (PoET) }\end{array}$ & 70000 & NO & $\begin{array}{c}\text { Relies on } \\
\text { Intel SGX } \\
\text { trusted } \\
\text { execution } \\
\text { environment } \\
\mathrm{f}<\mathrm{n} / 2\end{array}$ & $\begin{array}{l}\text { 63\% Python, } \\
\text { High } \\
\text { development } \\
\text { activity } \\
\text { Apache Version } \\
2.0\end{array}$ & Hyperledger project \\
\hline $\begin{array}{c}\text { Fabric } \\
--- \\
\text { https://github.com/hy } \\
\text { perledger/fabric }\end{array}$ & $\begin{array}{l}\text { PBFT consensus. } \\
\text { Uses Apache kafka and } \\
\text { zookeeper, depending on } \\
\text { the version }\end{array}$ & $\mathrm{n} / \mathrm{a}$ & YES & $\begin{array}{l}\text { High degrees } \\
\text { of } \\
\text { confidentialit } \\
\text { y, resilience, } \\
\text { flexibility, } \\
\text { scalability }\end{array}$ & $\begin{array}{c}86 \% \text { Go } \\
\text { active } \\
\text { development } \\
\text { Apache } 2.0\end{array}$ & Hyperledger project \\
\hline $\begin{array}{c}\text { Burrow } \\
--- \\
\text { https://github.com/hy } \\
\text { perledger/burrow }\end{array}$ & $\begin{array}{c}\text { PoS } \\
\text { Tendermint consensus } \\
\text { engine }\end{array}$ & $\mathrm{n} / \mathrm{a}$ & YES & $\mathrm{n} / \mathrm{a}$ & $\begin{array}{l}98.9 \% \text { Go } \\
\text { Apache } 2.0\end{array}$ & Smart contracts on EVM \\
\hline $\begin{array}{c}\text { Monax } \\
--- \\
\text { https://github.com/mo } \\
\text { nax/monax } \\
\end{array}$ & PoS & $\mathrm{n} / \mathrm{a}$ & YES & $\mathrm{n} / \mathrm{a}$ & $\begin{array}{l}\text { 93.7\% Go } \\
\text { Low } \\
\text { development } \\
\text { activity } \\
\end{array}$ & $\begin{array}{l}\text { Oriented to finance, insurance, } \\
\text { logistics. Monax includes smart } \\
\text { contracts }\end{array}$ \\
\hline $\begin{array}{c}\text { Iota } \\
--- \\
\text { https://github.com/iot } \\
\text { aledger }\end{array}$ & $\begin{array}{l}\text { PoW with hashcash. } \\
\text { Hash function: Curl } \\
\text { Signature: SHA-3 and } \\
\text { Winternit zone-time } \\
\text { signature (W-OTS) }\end{array}$ & 800 & NO & $\begin{array}{l}\text { Works well in } \\
\text { dense graphs. } \\
\text { The security } \\
\text { level is } \\
\text { unclear. }\end{array}$ & $\begin{array}{l}\text { Java } \\
\text { Iota Reference } \\
\text { Implementation } \\
\text { (IRI) }\end{array}$ & $\begin{array}{l}\text { Oriented to IoT } \\
\text { Based on Tangle (uses DAG rather } \\
\text { than the usual chain). Scalable, no } \\
\text { transaction fees, data integrity and } \\
\text { quantum-computing protection. }\end{array}$ \\
\hline $\begin{array}{c}\text { Corda } \\
--- \\
\text { https://github.com/cor } \\
\text { da/corda }\end{array}$ & $\begin{array}{c}\text { Notary infrastructure to } \\
\text { validate uniqueness and } \\
\text { sequencing of transactions, } \\
\text { no global broadcast } \\
\text { BFT-SMaRt } \\
\end{array}$ & $\mathrm{n} / \mathrm{a}$ & YES & $\mathrm{f}<\mathrm{n} / 3$ & $\begin{array}{l}94.4 \% \text { Kotlin } \\
\text { Apache } 2.0\end{array}$ & $\begin{array}{c}\text { Hashed DAG } \\
\text { Rather than classic } \\
\text { It is not a classic blockchain }\end{array}$ \\
\hline
\end{tabular}


available: public (or permissionless) blockchains, consortium (or permissioned) blockchains, and private blockchains. The differences between those categories depend on restrictions regarding who can read/write transactions: every node, a predefined subset, or centralized by one organization. In the following sections, the permissioned blockchain will be taken into account because only nodes belonging to a district can transact energy directly. The distributor will identify the set of nodes entitled to write the energy losses in the transaction block, based on their geographic location.

\section{ENERGY BLOCKCHAIN APPLICATION TO MICROGRID}

As demonstrated in [3], the blockchain technology in the energy field has become a realistic perspective, in particular for energy districts. Nevertheless, from a technical perspective, the issue poses some important challenges that are analyzed in this paper. Firstly, it is well-known that an energy transaction between a generator and a load, that are located in two different nodes, in general does not correspond to the physical situation that appears when power flow tracing methods are applied. This is true both for High Voltage (HV) systems [9] and for small Medium Voltage (MV) and Low Voltage (LV) distribution grids, where the presence of DG with voltage support systems (so-named P-V nodes) gives place to a large circulation of reactive power.

After the virtual transaction is initiated, a technical feasibility check is executed, to verify whether the considered transaction is or not viable in the current system. At the same time, the impact on the loads fed at that moment is assessed. If the technical verification gives a positive result and the buyer has sufficient credit, the physical transaction is started. Referring to Fig. 1, the energy transaction from time $t-2$ to $t+1$ (transaction $k$ ) implies that a generator sells power to a load. After some time, at $t-1$, a new transaction $(k+1)$ starts and the network sees a new energy flow between another generator and another load. If the tracing algorithm indicates a superposition of the paths between generators and loads, this transaction causes a nonlinear coupling of flows during time interval $\Delta t$ in Fig. 1.

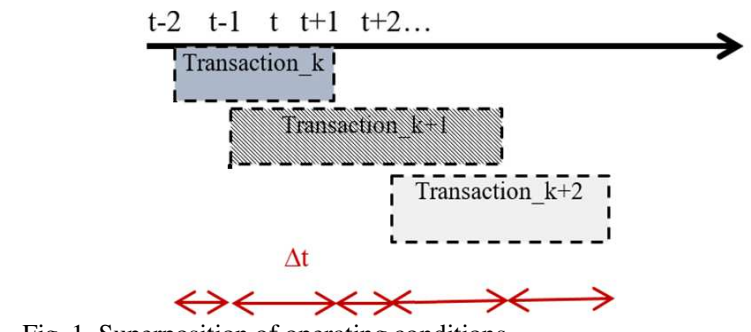

Fig. 1. Superposition of operating conditions.

As a consequence, a new power flow distribution can either increase or reduce the efficiency of the distribution system in terms of losses, although it is difficult to assess to what extent losses on a generic branch hosting two power flows from two generators can be shared among the loads. In the following, the methodology to carry out this analysis is outlined.

For every new energy transaction, based on the following working hypothesis: "for each scenario one of the generators supplying loads is considered as a slack bus and the other generator nodes are set as $P-V$ or $P-Q$ buses", the following steps are defined:

1) the set-points of the generators, that are not taking part to the new transaction and are considered as P-V or P-Q nodes, are fixed with a constraint on the reactive power production, according to the capability curve of each generator, and a value of active power fixed and equal to the previous transaction;

2) the load is increased of a specified amount (in $\mathrm{kW}$ );

3 ) the power flows tracing is performed and the contribution of each generator to loads and active energy losses in each branch is identified;

4) the increase/reduction of energy losses for the new energy transaction is quantified;

5) energy losses are attributed to each energy transaction, following the method described in Section IV.

As far as it concerns the first step, generators nodes not involved in the energy transaction are either considered as P-V or P-Q buses, given that DG units are either grid forming or grid supporting units [21]. Instead, the generator node involved in the new energy transaction is considered as a slack bus, in order to impose on it the supply also of the losses variation caused by the new transaction.

These energy losses are also included in the blockchain, as shown in Fig 2, where $n_{i}$ is the nonce of the i-th block and $\mathrm{TS}_{\mathrm{i}}$ is the timestamp. Energy losses are added to the corresponding intended transactions. Bundles of transactions and losses are signed in blocks, which are protected from tampering by the chained hash values. Additionally, losses vary also within transactions. Indeed, the overlap in time of multiple transactions generates heterogeneous operating conditions for any of the segments indicated by red arrows in Fig. 1.

The computation of energy losses proposed in this paper has to be run by a central entity, and we assign this role to selected nodes belonging to the DSO (Distributed System Operator), which continue balancing voltage points and maintaining the distribution network. For this reason, from the possible options listed in Table I, we take into special account permissioned blockchains. DSOs validate transactions, compute the power flow algorithm, provide energy losses, include them to transaction segments.

A fully-distributed approach where nodes run a distributed power flow algorithm is another possible option not explored in the present work.

As outlined above, one of the most important steps of the procedure is the power flow tracing. Active power flow tracing methodologies are well-established tools for analyzing power transmission networks [9]-[11], [22]-[35]. They, indeed, allow the assessment of the contribution of each generator to the branches power flows on each line. These methods are applied after the solution of the load flow problem. Therefore, for each experiment, a hypothesis about generation and load nodes should be done as first step. Extensive literature is available on the blockchain technology and on power flow tracing separately but their combination is lacking. 


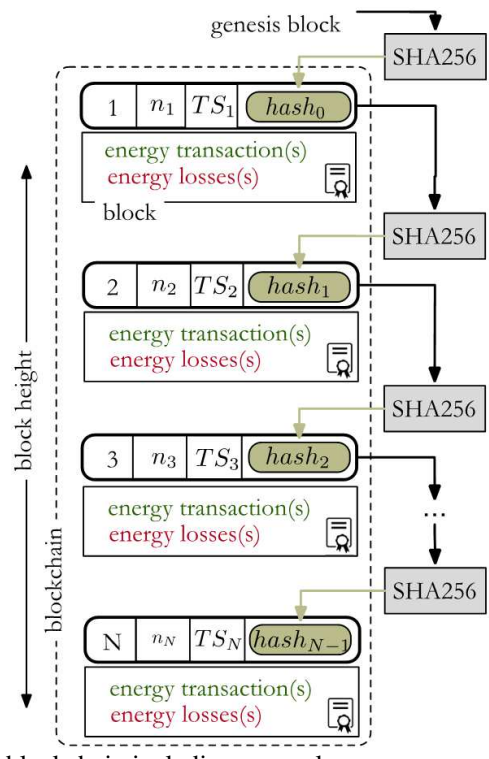

Fig. 2. The energy blockchain including power losses.

On one hand, the blockchain is studied in its relations with the components of microgrid energy markets: microgrid setup, grid connection, information system, market mechanism, pricing mechanism, energy management trading system and regulation [3]. This paper considers the missing component in the list, the technical handling of the grid.

On the other hand, the state of the art in power systems shows many papers describing the power flow tracing algorithms considering the proportional sharing assumption.

Fig. 3 shows a simple example for explaining the application of this principle. The contribution $P_{m}(j)$ from the ingoing power flow in a given branch " $j$ " to the outgoing power flow in the branch " $m$ " is proportional to the ratio of the power flow in " $j$ " and the total power flow through the node " $i$ " [22].

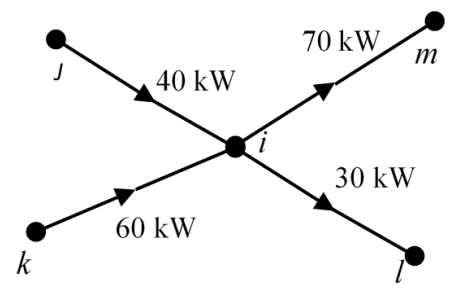

Fig. 3. Example of proportional sharing assumption.

Therefore, with reference to Fig. 3, the following relation can be written:

$$
\begin{aligned}
& P_{m}(j)=\frac{40}{40+60} 70=28 \mathrm{~kW} ; P_{m}(K)=\frac{60}{40+60} 70=42 \mathrm{~kW} \\
& P_{l}(j)=\frac{40}{40+60} 30=12 \mathrm{~kW} ; P_{l}(K)=\frac{60}{40+60} 30=18 \mathrm{~kW}
\end{aligned}
$$

with $P_{m}(j), P_{l}(j), P_{m}(k), P_{l}(k)$ representing the contribution of the ingoing power flows (in branches " $j$ " and " $k$ ") to the outgoing power flows (in branches " $m$ " an "l"). The coefficients:

$$
\alpha_{g e n(j-i)}^{i}=\frac{40}{40+60} ; \alpha_{g e n(k-i)}^{i}=\frac{60}{40+60}
$$

account for the contribution of the power flows in branches " $j$ " and " $k$ " to the power flows in branches " $m$ " an " $l$ ". Vice versa, the coefficients:

$$
\alpha_{\text {load }(i-m)}^{i}=\frac{70}{70+30} ; \alpha_{\text {load }(i-l)}^{i}=\frac{30}{70+30}
$$

account for the contribution of the power flows in branches " $m$ " and " $l$ " to the power flows in branches " $j$ " an " $k$ ".

The proportional sharing assumption has been widely employed in many analytical approaches for power flows tracing [19]-[35], although, generally, it was referred to the evaluation of the contribution of each generator to a branch power flow and not to the identification of the power losses allocated to a single transaction. All the referenced methods need the following input data as a result of the load flow calculation: power consumption of every load, power production of every generator, power flow in every line and transformer (being both of them seen as branches of a graph representation of the grid). The proportional sharing assumption allows to identify precisely what amount of power flow in each branch comes from a given generator and what amount of power flow (coming from a generator) goes to a given load. The tracing methodology allows to put a 'tag' on the active and reactive power flows, and to identify the physical paths connecting generators and loads. It must be underlined that the traced paths are identified based on the physical power flows in the network, while the transactions paths are based on the virtual blockchain smart contracts which do not reflect, in general, the technical frame of power distribution.

\section{LOSSES ATTRIBUTION ASSESSMENT}

As it can be easily understood, even if a generator and a load are in close contact, in general the injection of power from the generator will not directly supply the load. While this fact in the current energy market has little or no influence in the determination of energy distribution costs, it becomes relevant in a smaller context where the energy is exchanged between nearby nodes and peers. The attribution of power losses to a given energy transaction is, indeed, not straightforward. Moreover, voltage profiles, reactive flows and power losses are modified at each transaction for all nodes connected to the microgrid. Currently, the 'so called' distribution costs account for a given percentage of the energy tariff and are determined largely based on the amount of energy consumed per year. In this way, if consumption stays below a given threshold, distribution costs are low valued, otherwise their increase is not linear, penalizing more and more larger consumptions. The authors believe that this way of managing distribution costs is not suitable for $\mathrm{P} 2 \mathrm{P}$ transactions, since, as it will be cleared out by the proposed examples, the effect of each transaction is significant for all nodes of the grid (especially in islanded systems or when many voltage-supporting distributed generators are connected to the grid).

Moreover, the production capacity of DG is significantly affected by the large reactive power flows that may appear in the microgrid. Another issue to be accounted for is that the 
losses and the reactive support provided by generators 'depend' on the underlying physical situation. Moreover, although distribution charges can be exactly computed in each branch, they cannot be exactly attributed to contemporaneous transactions insisting on the same path, due to the non linear coupling of flows on the same branch deriving from different generators [29]. Therefore, the authors believe that the straightforward application of the proportional sharing rule to for power losses attribution assessment is not the best way to manage distribution costs.

In what follows, a proposal for losses attribution to energy transactions through two different indicators is given.

\section{A. Losses attribution}

With reference to the situation depicted in Fig. 4, in the following, an expression is derived to identify the amount of power losses that can be attributed to a generic transaction between a generic generator upstream nodes $j$ or $k$ and a generic load downstream nodes $l$ or $m$.

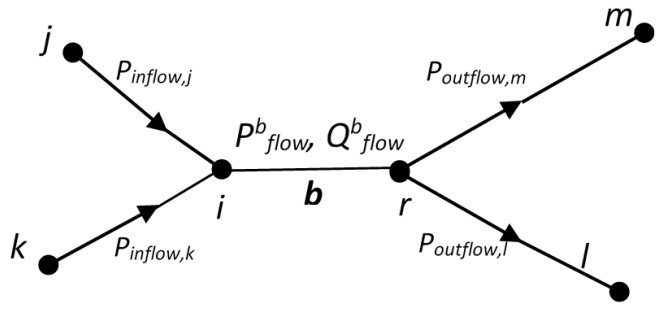

Fig.4. Losses partition on branch $b$.

The power flow $P_{b \text {,flow }}$ in branch $b$ (branch $i-r$ ) can be expressed as:

$$
P_{b, \text { flow }}=\sum_{h=1}^{n L} P_{\text {Load }, h}+P_{\text {Load }, r}+\sum_{h=1}^{n L} \Delta P_{b r, h}+\Delta P_{b}
$$

where:

$P_{\text {Load }, h}$ is the active power demand of the $h$-th of the $n_{L}$ loads downstream the branch $b$;

$P_{\text {Load }, r}$ is the active power demand of the load supplied by the branch $b$ at its ending bus $r$;

$\sum_{h=1}^{n L} \Delta P_{b r, h}$ is the term expressing the power losses due to the $n_{L}$ loads downstream the branch $b$;

$\Delta P_{b}$ is the term expressing the power losses in the branch $b$.

Referring to Fig. 4, the following relation can be written:

$$
P_{\text {outflow }, m}+P_{\text {outflow }, l}=\sum_{h=1}^{n L} P_{\text {Load }, h}+\sum_{h=1}^{n L} \Delta P_{b r, h}
$$

It is well known that in the generic branch $b$, with series impedance $\dot{Z}_{b}=R_{b}+j X_{b}$, the power losses can be expressed as a function of the sending bus voltage $V_{i}$ and of the active and reactive power flows in the branch as:

$$
\Delta P_{b}=\frac{R_{b}}{V_{i}^{2}}\left(P_{b, \text { flow }}^{2}+Q_{b, \text { flow }}^{2}\right)
$$

The power flow tracing provides coefficients $\alpha_{G i}^{i}$ for attributing part of the power flow on branch $b$ to the generic generators upstream nodes $j$ and $k$ feeding the branch $b$.
Assuming that $n_{G}$ generators are connected to the network, the following equations can be written:

$P_{b, \text { flow }}=\alpha_{G 1}^{i} P_{b, \text { flow }}+\alpha_{G 2}^{i} P_{b, \text { flow }}+\ldots+\alpha_{G n G}^{i} P_{b, \text { flow }}$

$Q_{b, \text { flow }}=\alpha_{G 1}^{i} Q_{b, \text { flow }}+\alpha_{G 2}^{i} Q_{b, \text { flow }}+\ldots+\alpha_{\text {GnG }}^{i} Q_{b, \text { flow }}$ (7)

with: $1=\alpha_{G 1}^{i}+\alpha_{G 2}^{i}+\cdots+\alpha_{G n G}^{i}$.

The generic coefficient $\alpha_{G i}^{i}$ equals the product of coefficients like $\alpha_{g e n}^{h}$, as defined above, and is calculated for all $h$ branches connecting the generic sending bus $i$ of branch $b$ to the generator node supplying it:

$$
\alpha_{G i}^{i}=\Pi_{h} \alpha_{g e n}^{h}
$$

Considering equations (7), equation (6) in case of $n_{G}=2$ can be rewritten as:

$$
\begin{aligned}
& \Delta P_{b}=\frac{R_{b}}{V_{i}^{2}}\left[\alpha_{G 1}^{i}{ }^{2}\left(P_{b, \text { flow }}^{2}+Q_{b, \text { flow }}^{2}\right)+\alpha_{G 2}^{i}{ }^{2}\left(P_{b, \text { flow }}^{2}+\right.\right. \\
& \left.\left.Q_{b, \text { flow }}^{2}\right)+4 \alpha_{G 1}^{i} \alpha_{G 2}^{i}\left(P_{b, \text { flow }}^{2}+Q_{b, \text { flow }}^{2}\right)\right]=\alpha_{G 1}^{i}{ }^{2} \Delta P_{b}+ \\
& \alpha_{G 2}^{i{ }^{2} \Delta P_{b}}+4 \alpha_{G 1}^{i} \alpha_{G 2}^{i} \Delta P_{b}
\end{aligned}
$$

In the general case of $n_{G}$ generators, equation (9) becomes:

$$
\Delta P_{b}=\sum_{h=1}^{n G} \alpha_{G h}^{i{ }^{2}} \Delta P_{b}+2 \sum_{\substack{j=1 \\ k=1 \\ j \neq k}}^{n G} \alpha_{G j}^{i} \alpha_{G k}^{i} \Delta P_{b}
$$

where $\alpha_{G h}^{i}{ }^{2} \Delta P_{b}$, is the amount of losses attributable for sure to generator $G_{h}$ among those supplying branch $b$. On the contrary, the last term in (10) involves combined contributions of couples of generators and it is not possible for this amount to identify the percentages of contribution of each generator. It can be assumed that this amount, for which it is not clear the allocation, can be equally divided among the $n_{G}$ generators. Therefore, it can be written that the losses in branch $b$ attributable to generator $G_{i},\left(\Delta P_{b}\right)_{G i}$, are:

$$
\left(\Delta P_{b}\right)_{G i}=\left(\alpha_{G i}^{i^{2}}+\frac{2}{n_{G}} \sum_{\substack{j=1 \\ k=1 \\ j \neq k}}^{n G} \alpha_{G j}^{i} \alpha_{G k}^{i}\right) \Delta P_{b}
$$

If a generic energy transaction starts between load Loadz and generator $G_{i}$, equation (11) can still be used in the following way to assess the amount of losses that can be allocated to the transaction. Again, a proportional sharing rule will be considered using the coefficients $\alpha_{\text {load }}$ above defined. In this case, the coefficient to be used to assess the amount of losses allocated to a transaction that involves Loadz is attained by making the productory among all the coefficients $\alpha_{\text {load }}$ connecting the branch $b$ to the Loadz. As a result, the coefficient $\alpha_{L z}^{i}=\Pi_{h} \alpha_{\text {load }}^{h}$ accounts for the partition of power losses with regard to the load downstream branch $b$. Therefore, equation (12), when referred to the energy transaction between generator $G i$ and Loadz becomes: 


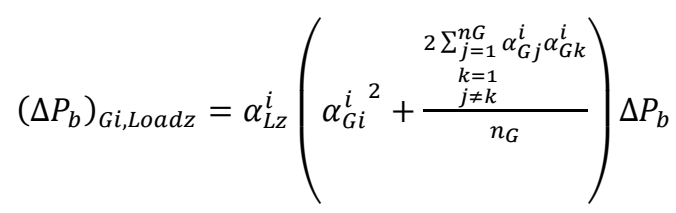

In order to consider only the increment of power $P_{\text {Loadz }}^{\Delta}$ required by Loadz during the energy transaction, the coefficient $\frac{P_{\text {Loadz }}^{\Delta}}{\text { Loadz }}$ must be introduced. Therefore, the final expression accounting for the allocation of losses on branch $b$ to the transaction between generator $G i$ and Loadz for an amount $P_{\text {Loadz }}^{\Delta}$ is:

$$
\begin{aligned}
& (\Delta P)_{G i, \text { Loadz }}=\sum_{b=1, n b}\left(\Delta P_{b}\right)_{G i, \text { Loadz }}= \\
& \quad=\sum_{b=1, n b} \alpha_{L z}^{i}\left(\alpha_{G i}^{i^{2}}+\frac{2}{n_{G}} \sum_{\substack{j=1 \\
k=1 \\
j \neq k}}^{n G} \alpha_{G j}^{i} \alpha_{G k}^{i}\right) \Delta P_{b} \cdot \frac{P_{L o a d z}^{\Delta}}{\operatorname{Loadz}}
\end{aligned}
$$

In this expression, $n b$ is the set of branches involved in the transaction and discovered via power flow tracing. In the following the index given by (13) will be indicated as PSR (Proportional Sharing Rule index).

Another simplified index accounting for losses attribution to each transaction can be defined. In distribution systems, losses are typically quantified in terms of a percentage of purchases [36]. Therefore, the following simplified method is set up for allocating losses to a transaction involving a given load increase. Given a transaction between generator $G_{i}$ and $L o a d z$ assume that $P_{L o a d z}^{\Delta}$ is purchased from $G_{i}$. The losses that can be attributed to this transaction according to a more simple expression, $(\Delta P)_{G i, L o a d z}$, are:

$$
(\Delta P)_{\text {Gi,Loadz }}=\Delta P_{\text {tot }} \cdot\left(\frac{P_{\text {Load } z}^{\Delta}}{\text { Load,tot }}\right)
$$

where Load,tot is the summation of all loads supplied by the network considering the increment due to the current transaction and $\Delta P_{t o t}$ is the overall network losses. In the following, the index given by (14) will be indicated as Global. This index is substantially different from PSR because it does not consider the actual path between generator $G_{i}$ and load Loadz. In general, it gives a less accurate estimation of the power losses but, at the same time, reduces the overall computational effort. In the application section, the two indices, PSR and Global, are compared in the different considered scenarios.

\section{APPLICATION}

The aim of this application section is to show how to allocate microgrid's power losses to each transaction using the methodologies described in section IV.

A 9-bus MV microgrid with three lines, three generators DG2, DG3 and DG4, and six loads, is considered. Table II reports the electrical features of the MV network. The parameter settings were extrapolated from real data in [37][38].
Two different scenarios are simulated. In the first scenario (Scenario 1), the microgrid is connected to the main grid at bus 1 and three photovoltaic (PV) generators with energy storage, are connected to buses 2, 3 and 4. Each producer satisfies the local demand of active energy and manages the rest of its production by the blockchain mechanism thanks to energy transaction with the passive users of the microgrid. The generators take part to the voltage regulation service producing also reactive power, according to specific technical rules (for example in Italy the mandatory technical rule is the Italian Standard CEI 0-16) [38]. In the second scenario (Scenario 2)

the microgrid is operated in island mode without any power exchange with the main grid and is supplied by three small DG units at buses 2, 3 and 4. The generators produce both real and reactive power to meet loads and power losses in the microgrid.

In both the scenarios, three energy transactions take place and partially overlap between instants $t_{1}$ and $t_{4}$. In the following, the two scenarios are simulated and compared in terms of losses allocation, considering a P2P energy transaction model like that based on the blockchain technology.

TABLE II. ElECTRICAL FEATURES OF THE NETWORK

\begin{tabular}{|c|c|c|c|}
\hline Sending bus & Ending bus & $\mathbf{R}[\boldsymbol{\Omega}]$ & $\mathbf{X}[\boldsymbol{\Omega}]$ \\
\hline 1 & 5 & 0.6076 & 0.098 \\
\hline 1 & 7 & 0.868 & 0.14 \\
\hline 1 & 2 & 0.4487 & 0.091 \\
\hline 2 & 8 & 0.641 & 0.13 \\
\hline 3 & 6 & 1.302 & 0.21 \\
\hline 5 & 3 & 0.868 & 0.14 \\
\hline 7 & 4 & 1.302 & 0.21 \\
\hline 8 & 9 & 0.9615 & 0.195 \\
\hline
\end{tabular}

A. Scenario 1

In this scenario, the generation units contribute to voltage regulation providing reactive power during energy transactions, according to the limits imposed by the Italian Standard CEI 016. The following energy transactions take place between instants $t_{1}$ and $t_{4}$ : $a$. between instants $t_{1}$ and $t_{4}$, the user Load7 buys $350 \mathrm{~kW}$ from DG2; b. between instants $t_{2}$ and $t_{4}$, the user Load5 buys $250 \mathrm{~kW}$ from DG4; c. between instants $t_{3}$ and $\mathrm{t}_{4}$, the user Load9 buys $183 \mathrm{~kW}$ from DG3.

The power flow solution and the tracing produce the situation depicted in Figures 5-7.

The three figures show that each virtual transaction influences the whole microgrid, modifying the physical power flows and their partition with reference to the generators. As a result, the losses allocation to the generators changes along time as depicted in Fig. 8. It is worth to note that, in this scenario, each DG begins to inject power into the network right when the transaction involving it takes place.

The energy transactions modify the allocation of losses mostly for the main grid for which losses are reduced of $11 \%$ and for generator DG2 for which losses increase of $26 \%$. PSR and Global values are reported in Table III for each transaction as well as the total losses in the whole network. 


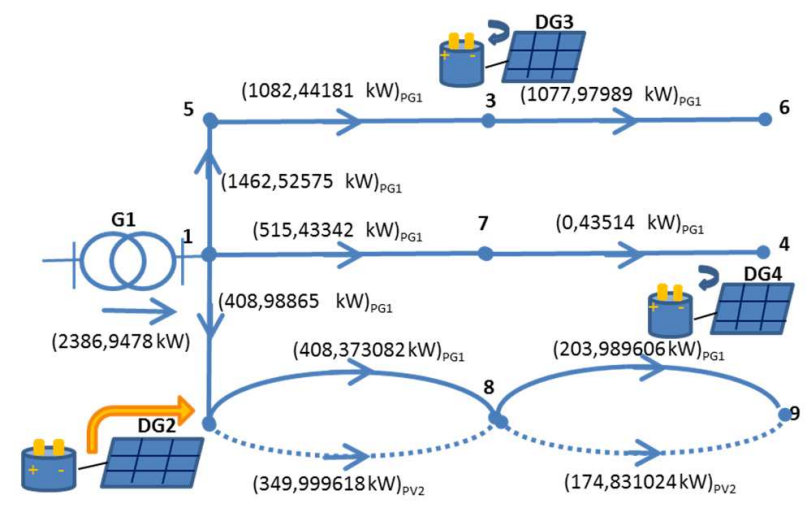

Fig. 5. Power flow tracing. Scenario $1-t_{1}-t_{2}$.

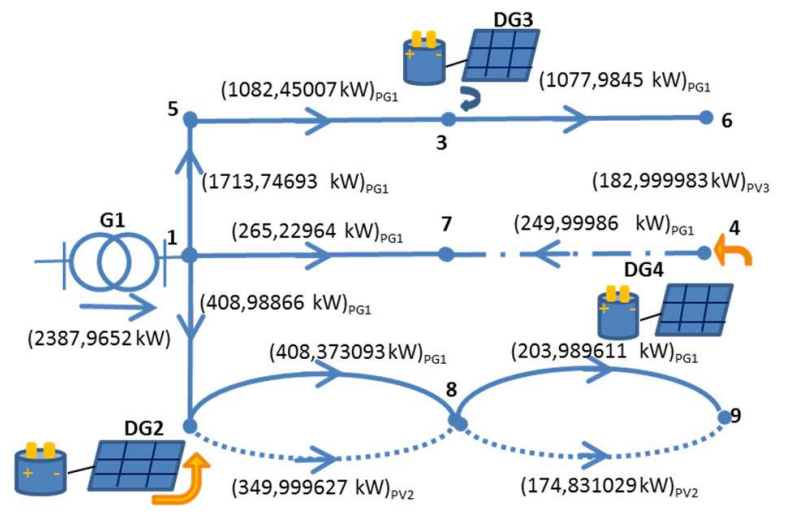

Fig. 6. Power flow tracing. Scenario $1-\mathrm{t}_{2}-\mathrm{t}_{3}$.

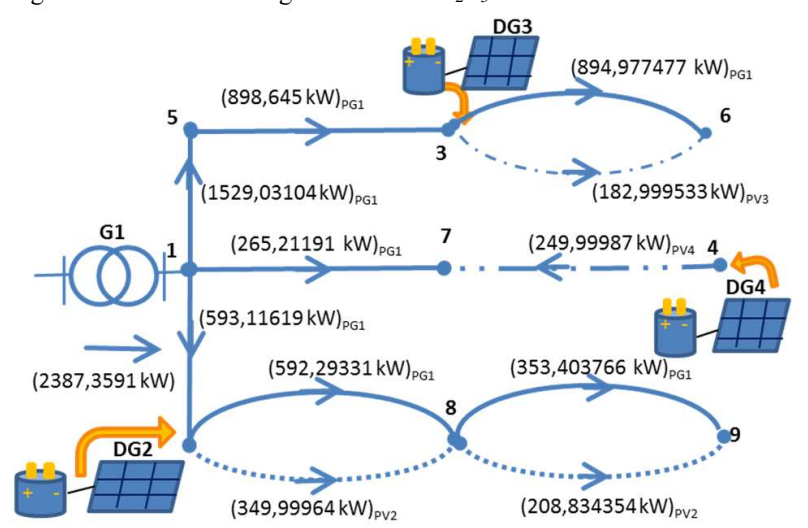

Fig. 7. Power flow tracing. Scenario $1-\mathrm{t}_{3}-\mathrm{t}_{4}$.

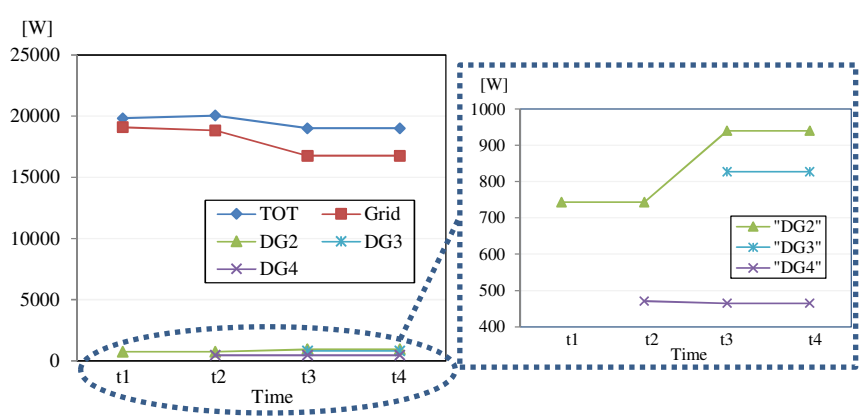

Fig. 8. Power losses allocation to generators - Scenario 1.- Grid refers to the substation.
TABLE III. LOSSES ATTRIBUTION TO TRANSACTIONS. SCENARIO 1.

\begin{tabular}{|c|c|c|c|c|c|}
\cline { 4 - 6 } \multicolumn{2}{c|}{$\begin{array}{c}\text { Total } \\
\text { losses } \\
{[\mathrm{W}]}\end{array}$} & $\mathrm{t}_{1}-\mathrm{t}_{2}$ & $\mathrm{t}_{2}-\mathrm{t}_{3}$ & \multirow{2}{*}{$\mathrm{t}_{3}-\mathrm{t}_{4}$} \\
\hline \multirow{2}{*}{ trans1 } & PSR [W] & \multirow{2}{*}{19828.89} & 1094.06 & 805.18 & 798.46 \\
\cline { 2 - 5 } & Global [W] & & 2262.08 & 2114.77 & 1899.39 \\
\hline \multirow{2}{*}{ trans2 } & PSR [W] & \multirow{2}{*}{20048.17} & - & 944.98 & 876.58 \\
\cline { 2 - 6 } & Global [W] & - & 1510.55 & 1356.71 \\
\hline \multirow{2}{*}{ trans3 } & PSR [W] & \multirow{2}{*}{18999.50} & - & - & 271.11 \\
\cline { 2 - 6 } & Global [W] & & - & - & 993.11 \\
\hline
\end{tabular}

\section{B. Scenario 2}

In this scenario, the microgrid is isolated from the main grid and the generation units fully contribute to voltage regulation providing reactive power during energy transactions. The following energy transactions take place between instants $t_{1}$ and $t_{4}$ : a. between instants $t_{1}$ and $t_{4}$, the user Load7 buys $350 \mathrm{~kW}$ from DG2; b. between instants $t_{2}$ and $t_{4}$, the user Load 5 buys $250 \mathrm{~kW}$ from DG3; c. between instants $t_{3}$ and $t_{4}$, the user Load9 buys $183 \mathrm{~kW}$ from DG1. The power flow solution and the following power flow tracing produce the situation depicted in Figures 9-11.

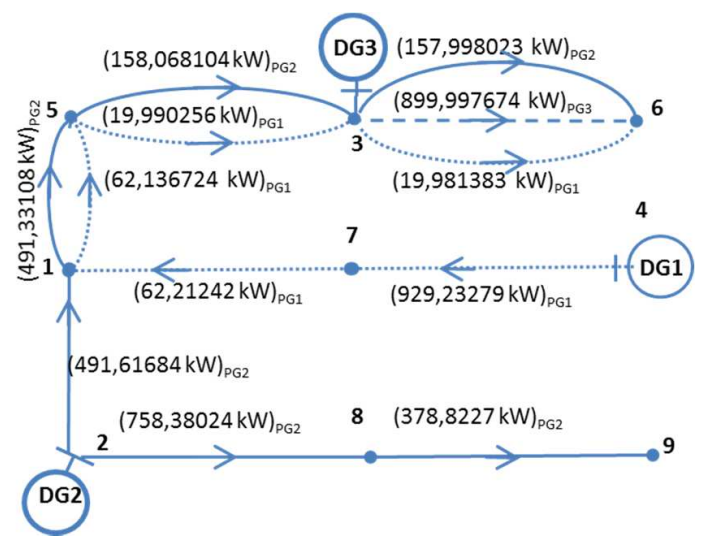

Fig. 9. Power flow tracing. Scenario $2-\mathrm{t}_{1}-\mathrm{t}_{2}$.

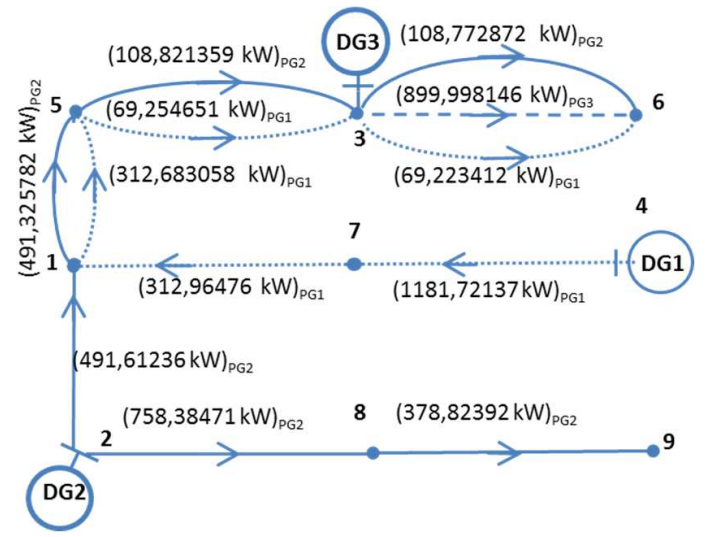

Fig 10. Power flow tracing. Scenario $2-t_{2}-t_{3}$.

Losses allocation to generators changes along time as depicted in Fig. 12. The energy transactions in the microgrid modify the losses allocation mostly for generator DG4, for which losses increase of more than $60 \%$ in time interval $t_{2}-t_{3}$ with respect to the previous time interval, and of $50 \%$ overall. 
For generator DG3, losses increase of $18 \%$ overall.

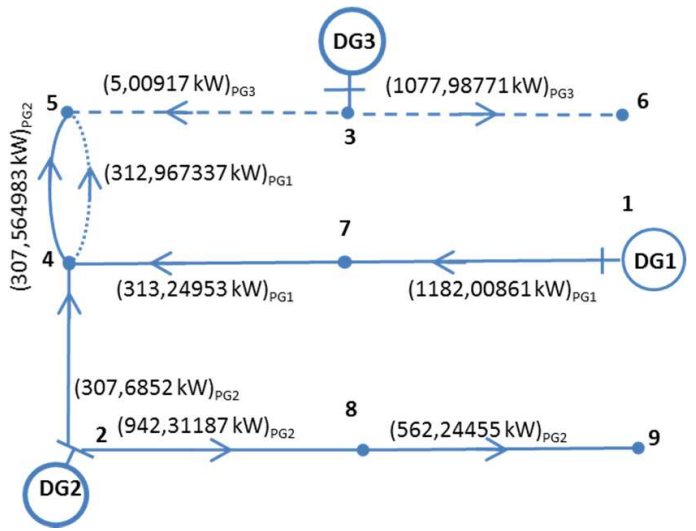

Fig. 11. Power flow tracing. Scenario $2-t_{3}-t_{4}$.

PSR and Global values for each transaction are reported in Table IV, as well as the total losses in the microgrid.

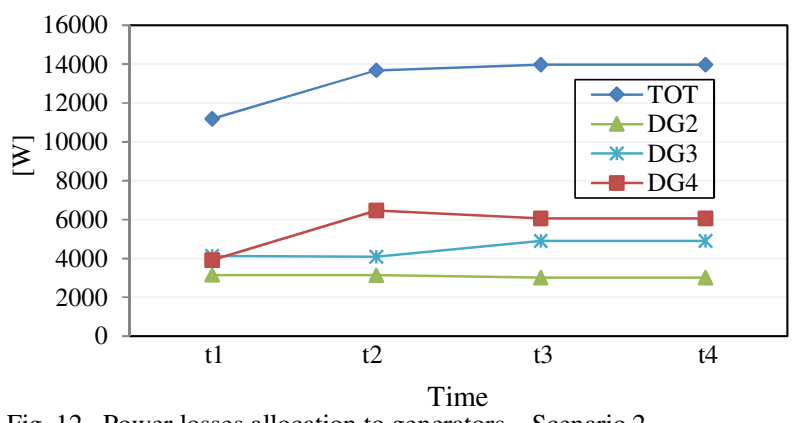

Fig. 12.. Power losses allocation to generators - Scenario 2.

TABLE IV. LOSSES ATTRIBUTION TO TRANSACTIONS. SCENARIO 2.

\begin{tabular}{|c|c|c|c|c|c|}
\hline & & $\begin{array}{c}\text { Total losses } \\
\text { [W] }\end{array}$ & $t_{1}-t_{2}$ & $\mathrm{t}_{2}-\mathrm{t}_{3}$ & $t_{3}-t_{4}$ \\
\hline \multirow{2}{*}{$\operatorname{trans} 1$} & PSR [W] & \multirow{2}{*}{11198.55} & 1390.60 & 1610.77 & 1611.12 \\
\hline & Global [W] & & 1277.53 & 1443.78 & 1396.95 \\
\hline \multirow[b]{2}{*}{$\operatorname{trans} 2$} & PSR [W] & \multirow[b]{2}{*}{13687.12} & - & 3920.48 & 2755.90 \\
\hline & Global [W] & & - & 1031.27 & 997.82 \\
\hline \multirow{2}{*}{$\operatorname{trans} 3$} & PSR [W] & \multirow{2}{*}{13973.57} & - & - & 610.08 \\
\hline & Global [W] & & - & - & 730.40 \\
\hline
\end{tabular}

\section{Discussion}

Some interesting outcomes are obtained by comparing Scenarios 1 and 2. Comparing Figures 8 and 12, it can be observed that overall losses are generally lower in the second scenario and that they increase as the load increases. This behavior depends on the capability of voltage support from the generation units in Scenario 2 and, consequently, on the different voltages distribution (generally higher).

Looking at Tables III and IV, it can be observed that the Global index gets higher values as compared to PSR in Scenario 1, and lower values in Scenario 2. Being the Global index strongly correlated with the overall losses of the microgrid, it is more affected by the configuration of the network and less affected by the specific transaction. The Global index provides a very different assessment as compared to the more accurate PSR indicator. As an example, in the first scenario, where the grid in the third transaction supplies almost $80 \%$ of the overall load, the losses cost allocation appears very unfair using the Global index. Indeed, the index allocates to the transactions as a whole around $20 \%$ of the overall losses in line with the load increase hypothesized from $t_{1}$ to $t_{4}$. PSR keeps cost allocation more in line with the realistic weight the transactions have in the network (around 10\%). Scenario 1 indeed shows a decreasing trend for overall losses, therefore the effect of load increase is beneficial as shown by PSR. For this reason, the Global index could be used for attributing the power losses to the transaction in a simple way but accepting an inherently low precision.

Another important outcome of the study is that the presence of a connection to the main grid strongly affects the active and reactive power flows in the microgrid, since the transactions occurring in the microgrid are the same in the two considered scenarios. For this reason, the power losses estimated for the same transactions in presence and in absence of connections are very different. In particular, the absence of connection to the main grid gives rise to a different effort of the generation units for supporting the microgrid's voltage.

The variations of the indices in the two examined cases demonstrate the importance of attributing the power losses to each transaction for a correct evaluation of the energy exchange between generators and loads.

The block to be included in the blockchain will contain extra data regarding power losses. This information is available only at the end of each transaction segment, therefore at the end of each time interval, assuming, for the sake of simplicity an equal attribution of losses to the participating loads.

In Table V, an exemplary block of transactions is depicted, based on the results obtained in Table IV, in case of PSR evaluation mode.

TABLE V. LOSSES ATTRIBUTION TO TRANSACTIONS. SCENARIO 2.

\begin{tabular}{|c|c|c|c|}
\hline block ID i & Nonce $\mathrm{i}$ & Timestamp $t_{4}$ & $\mathrm{Hash}_{\mathrm{i}}$ \\
\hline \multicolumn{4}{|c|}{$\begin{array}{l}\text { Time: } \mathrm{t}_{2} \\
\text { From: DG2 to: Load7 Energy: }\left(\mathrm{t}_{2}-\mathrm{t}_{1}\right) * 350 \mathrm{kWh} \\
\text { From: Load7 to: DSO Losses: }\left(\mathrm{t}_{2}-\mathrm{t}_{1}\right) * 1.390 \mathrm{kWh}\end{array}$} \\
\hline \multicolumn{4}{|c|}{$\begin{array}{l}\text { Time: } \mathrm{t}_{3} \\
\text { From: DG2 to: Load7 Energy: }\left(\mathrm{t}_{3}-\mathrm{t}_{2}\right) * 350 \mathrm{kWh} \\
\text { From: Load7 to: DSO Losses: }\left(\mathrm{t}_{3}-\mathrm{t}_{2}\right) * 1.610 \mathrm{kWh} \\
\text { From: DG4 to: Load5 Energy: }\left(\mathrm{t}_{3}-\mathrm{t}_{2}\right) * 250 \mathrm{kWh} \\
\text { From: Load5 to: DSO Losses: }\left(\mathrm{t}_{3}-\mathrm{t}_{2}\right) * 3.920 \mathrm{kWh}\end{array}$} \\
\hline \multicolumn{4}{|c|}{$\begin{array}{l}\text { Time: } \mathrm{t}_{4} \\
\text { From: DG2 } \text { to: Load7 Energy: }\left(\left(\mathrm{t}_{4}-\mathrm{t}_{3}\right) * 350 \mathrm{kWh}\right. \\
\text { From: Load7 to: DSO Losses: }\left(\mathrm{t}_{4}-\mathrm{t}_{3}\right) * 1.61112 \mathrm{kWh} \\
\text { From: DG4 to: Load5 Energy: }\left(\mathrm{t}_{4}-\mathrm{t}_{3}\right) * 250 \mathrm{kWh} \\
\text { From: Load5 to: DSO Losses: }\left(\mathrm{t}_{4}-\mathrm{t}_{3}\right) * 2.75590 \mathrm{kWh} \\
\text { From: DG3 to: Load9 Energy: }\left(\mathrm{t}_{4}-\mathrm{t}_{3}\right) * 250 \mathrm{kWh} \\
\text { From: Load9 to: DSO Losses: }\left(\mathrm{t}_{4}-\mathrm{t}_{3}\right) * 0.61008 \mathrm{kWh}\end{array}$} \\
\hline
\end{tabular}

In relation to what seen in Fig. 1, the whole transaction is not computed for each couple of nodes but a different evaluation of transacted energy and losses and relevant attribution is provided for each segment in which the operating conditions do not change. 


\section{CONCLUSION}

This paper has presented the blockchain as a distributed tool that goes beyond the economic aspects of energy transactions and can be used to make distributed decisions for technical operations in microgrids. In particular, the proposed study focuses on the issue of energy losses tracking and attribution during the energy transaction and shows that including losses in the blockchain changes dramatically the timing of information gathering and the role of prosumers and distributors.

Indeed, in microgrids the intended power flows of P2P energy transactions do not match the physical situation, which is highly constrained by the technical issue. Reactive flows strongly affect the grid's operation, especially when local generators provide the voltage regulation service. Moreover, the missing match between virtual and physical transactions makes difficult a correct power losses allocation. For this reason, the authors have proposed two indices for power losses allocation. The proposed PSR index is able to give a more accurate evaluation of the power losses to be associated with the energy transaction between a specific couple generator/load. The Global index, on the contrary, gives only an approximate evaluation of the power losses, but is very simple to calculate. Losses allocation in energy transactions is not a straightforward issue, due to the nonlinear coupling of mutual terms. In this paper, it is argued that mutual terms appearing in the power losses expression could be equally shared among transactions that are active on a given branch.

Moreover, in the present work, we considered prosumers as negotiators of energy transactions and the distributor as an entity for computing losses, thanks to its knowledge on the whole distribution network. Eventual economic rewards for the distributor, for compensating the cost of network maintenance, could be additionally included in the blockchain. However, their computation algorithm would be defined by regulatory bodies and, therefore, is out of the scope of the present paper. Further extensions of this work will investigate the optimal reactive power flow in order to limit circulation of reactive flow and still guarantee a good power quality. Also, other ancillary services provision will be considered and a reward system will also be proposed. Finally, also the effect of changing operating condition on the upstream system will be investigated for further analysis of the proposed business model.

\section{REFERENCES}

[1] Gartner, "Top Trends in the Gartner Hype Cycle for Emerging

$$
\text { 2017", }
$$
available

http:/www.gartner.com/smarterwithgartner/top-trends-in-the-gartnerhype-cycle-for-emerging-technologies-2017/.

[2] P. Frøystad, and J. Holm, "Blockchain: Powering the Internet of Value", EVRY Labs, 2016.

[3] E. Mengelkamp, J.s Gärttner, K. Rock, S. Kessler, L. Orsini, and C. Weinhardt, "Designing microgrid energy markets: A case study: The Brooklyn Microgrid", Applied Energy, Vol. 210, 2018, pp. 870-880.

[4] J. Kang, R. Yu, X. Huang, S. Maharjan, Y. Zhang, and E. Hossain, "Enabling Localized Peer-to-Peer Electricity Trading Among Plug-in Hybrid Electric Vehicles Using Consortium Blockchains", IEEE Transactions on Industrial Informatics, Vol. 13, Issue 6, 2017, pp. 3154 3164
[5] H. K. Trabish, "Public purpose microgrids: Mixed-ownership models spur utility investment in growing sector" available athttp://www.utilitydive.com/news/public-purpose-microgrids-mixedownership-models-spur-utility-investment-i/425296/.

[6] Z. Li, J. Kang, R. Yu, D. Ye, Q. Deng, and Y. Zhang, "Consortium Blockchain for Secure Energy Trading in Industrial Internet of Things", IEEE Transactions on Industrial Informatics, Vol. PP, Issue 99, 2017, DOI: 10.1109/TII.2017.2786307.

[7] M. E. Peck, and D. Wagman, "Energy trading for fun and profit buy your neighbor's rooftop solar power or sell your own-it'll all be on a blockchain”, IEEE Spectrum, Vol. 54, Issue 10, 2017, pp. 56-61.

[8] E. Munsing, J. Mather, and S. Moura, "Blockchains for decentralized optimization of energy resources in microgrid networks," 2017.

[9] L. Dusonchet, S. Favuzza, M. G. Ippolito, F. Massaro, and G. Paternò, "Numerical implementation of active power flow tracing methods: practical implications on transmission networks and DR programs support", 2015 IEEE 15th Intl. Conf. on Environment and Electrical Engineering, pp. 531 - 536, 10-13 June 2015, Rome (Italy).

[10] P. M. Sotkiewicz, and J. M. Vignolo, "Allocation of Fixed Costs in Distribution Networks with Distributed Generation", IEEE Transactions on Power Systems, Vol. 21, Issue 2, 2006, pp. 639-652.

[11] C. L.T. Borges, and D. M. Falcao, "Optimal distributed generation allocation for reliability, losses, and voltage improvement", Electrical Power and Energy Systems, Vol. 28, 2006, pp. 413-420

[12] A. Kosba, A. Miller, E. Shi, Z. Wen, C. and Papamantho, "Hawk: The blockchain model of cryptography and privacy-preserving smart contracts", Security and Privacy (SP), 2016 IEEE Symposium on, pp. 839-858, 23-25 May 2016, San Jose (USA).

[13] G. Zyskind, O. Nathan, and A. Pentland, "Decentralizing Privacy: Using Blockchain to Protect Personal Data", 2015 IEEE Security and Privacy Workshops, pp. 180-184, 21 May 2015, San Jose (USA).

[14] Q. Lu, and Xiwei Xu, "Adaptable Blockchain-Based Systems: A Case Study for Product Traceability", IEEE Software, Vol. 34, Issue 6, 2017, pp. 21-27.

[15] M. Swan, "Blockchain: Blueprint for a new economy", O'Reilly Media Inc., 2015.

[16] D. Tapscott, and A. Tapscott, "Blockchain Revolution: How the Technology Behind Bitcoin Is Changing Money, Business, and the World", Penguin Publishing Group, 2016.

[17] H. Gilbert, and H. Handschuh, "Security Analysis of SHA-256 and Sisters", Lecture Notes in Computer Science, Vol. 3006, Selected Areas in Cryptography, pp. 175-193, 2003.

[18] A. Back, "Hashcash-a denial of service counter-measure", Technical Report, 2002, available at: http://hashcash.org/papers/hashcash.pdf.

[19] C. Cachin and M. Vukolic, "Blockchain consensus protocols in the wild," CoRR, vol. abs/1707.01873v2, July 2017

[20] K. J. O'Dwyer and D. Malone, "Bitcoin mining and its energy footprint," in In Proceedings of the 2014 IET Irish Signals and Systems Conference, IET, 2014.

[21] X. Wang, J. Guerrero, Z. Chen, and F. Blaabjerg, "Distributed energy resources in grid interactive AC microgrids", 2nd IEEE International Symposium on Power Electronics for Distributed Generation Systems (PEDG) 2010, pp. 806-812, 16-18 June 2010, Hefei (China).

[22] J. Bialek, "Tracing the flow of electricity", IEE Proc. Generation, Transmission and Distribution, Vol. 143, n.4, 1996, pp. 313- 320.

[23] D. Kirschen, R. Allan, and G. Strnac, "Contributions of individual generators to loads and flows," IEEE Transactions on Power Systems,Vol. 12, n. 1, 2007, pp. 52-60.

[24] F.F. Wu, Y. Ni. and P. Wei, "Power transfer allocation for open accessusing graph theory - Fundamentals and applications in systems without loop flow", IEEE Transactions on Power Systems, Vol. 15, n. 3, 2000, pp.923-929.

[25] F.F. Wu, Y. Ni and P. Wei, "Load flow tracing in power system with circulation power", International Journal of Electrical Power \& Energy Systems, Vol. 24, n. 10, 2002, pp. 807-813.

[26] K. Xie, J. Zhou, and W. Li, "Analytical model and algorithm for tracing active power flow based on extended incidence matrix", Electric Power System Research, Vol. 79, n. 2, 2009, pp. 399-405.

[27] M.W. Mustafa, and H.Shareef, "A Comparison of Electric Power Tracing Methods Used in Deregulated Power Systems", IEEE International Power and Energy Conference, pp. 156-160, 28-29 November 2006, Putra Jaya (Malaysia).

[28] L. Dusonchet, S. Favuzza, M.G. Ippolito, and E. Riva Sanseverino, "A model for reactive power tracing by addition of fictitious nodal 
injections", Electric Power Systems Research, Vol. 83, n. 1, 2012, pp. 196-202.

[29] A. Augugliaro, L. Dusonchet, S. Favuzza, M.G. Ippolito, and E. Riva Sanseverino, "Influence of losses partition criteria on power flow tracing," IEEE Int Energy Conference and Exhibition, pp. 310-317, 9-12 September 2012, Florence (Italy).

[30] J.C. Peng, and H. Jiang, "Contributions of individual generators to complex power losses and flows. Fundamental theory," IEE ProceedingsGeneration, Transmission and Distribution, Vol. 149, n. 2, 2002, pp. 182 185.

[31] Z. Jing, and F. Wen, "Discussion on the Proving of Proportional Sharing Principle in Electricity Tracing Method," Transmission and Distribution Conference and Exhibition: Asia and Pacific, pp. 1-5, 14-18 August 2005, Dalian (China).

[32] D. Kirschen, and G. Strbac, "Tracing active and reactive power between generators and loads using real and imaginary currents," IEEE Transactions on Power Systems, Vol. 14, n. 4, 1999, pp. 1312-1319.

[33] Z. Ming, S. Liyng, L. Gengyin, and Y. Ni, "A novel power flow tracing approach considering power losses," IEEE International Conference onElectric Utility Deregulation, Restructuring and Power Technologies, vol. 1, pp. 355-359, 5-8 April 2004.

[34] F. Gubina, D. Grgic, and I. Banic, "A method for determining the generators' share in a consumer load," IEEE Transactions on Power Systems, Vol. 15, n. 4, 2000, pp. 1376-1381.

[35] S.P. Rosado, K. Abdel-Rahman, and N. Hadjsaid, "Tracing the path ofelectric power flow-a study for deregulated power systems," IEEE Power Engineering Society Winter Meeting, Vol. 3, pp. 1479-1484, January 2001

[36] J. Triplett, S.Rinell, and J. Foote, "Evaluating Distribution System Losses Using Data from Deployed AMI and GIS Systems", 2010 IEEE Rural Electric Power Conference (REPC), pp. C1 - C1-8, 16-19 May 2010, Orlando, Florida (USA)

[37] Andrea Butti. Title of Master Degree Thesis: Controllo predittivo di reti radiali con generazione distribuita. Tutor: Prof. Riccardo Scattolini. A.A. 2011-2012. Department of Electronics and Information, Politecnico di Milano

[38] Italian Technical Standard CEI 0-16, "Reference technical rules for the connection of active and passive consumers to the $\mathrm{HV}$ and MV electrical networks of distribution Company", July 2016

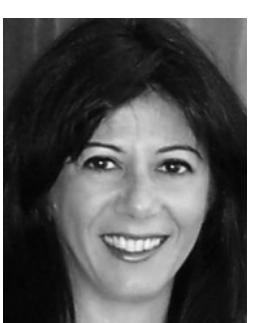

M. L. Di Silvestre is an Assistant Professor at the University of Palermo since 2000. She received the M.S. and $\mathrm{Ph} . \mathrm{D}$. degrees in electrical engineering from the University of Palermo in 1993 and 1998, respectively. Since 1999, she is with the DEIM of the University of Palermo. She was a Grant Holder from 1999 to 2000. Her research interests include analysis and modeling of complex grounding systems, optimization methods for electrical distribution systems design, operation, and planning, optimal power flow.

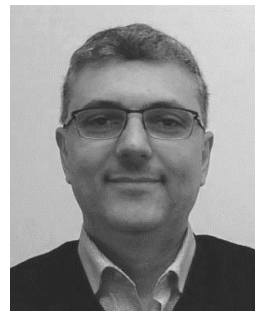

P. Gallo is an Assistant Professor at the University of Palermo since November 2010. He graduated with distinction in Electronic Engineering in July 2002 and received his $\mathrm{PhD}$ in 2015. He worked at CRES (Electronic Research Center in Sicily) until 2009 . His research activity is focused on wireless networks at the MAC layer and 802.11 extensions, cross layer solutions, indoor localization exploiting radio propagation and blockchain technology and its applications in smart grids, tracing and ecommerce. P. Gallo has contributed to several scientific papers and three patents and to several research projects.

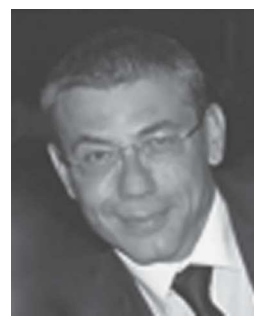

M. G. Ippolito is Full Professor of power systems with the University of Palermo since 2017. Since 2001, he has been locally responsible for several research projects in the field of power distribution development, distributed generation, electrical storage systems, and smart grids. $\mathrm{He}$ is currently the Coordinator of the M.Sc. Course in Electrical Engineering at the University of Palermo. In his areas of interest, he has authored or coauthored more than 150 technical papers published in international journals or presented at various national and international conferences. His main research interests include power systems analysis, optimal planning, and design and control of electrical distribution systems. In recent years, his research activities have included the integration of distributed generation and storage systems into electrical networks and smart grids.

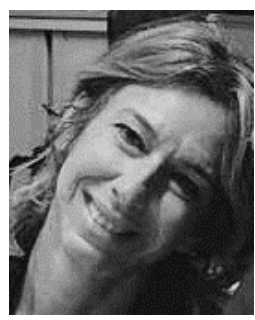

E. Riva Sanseverino is Associate Professor of Electrical Power Systems since 2002 at the University of Palermo and abilitated as Full Professor by the Italian Ministry of Education since 2013. Since 2002 she has been working at the DEIM of the University of Palermo. She is a scientific coordinator of various research projects with research organizations and companies. She is also responsible of various teaching cooperation agreements with foreign institutions. She authored more than 250 papers on international journals, edited books and book chapters and conference proceedings. She is the Rector's delegate of the European Charter and code for Researchers at the University of Palermo.

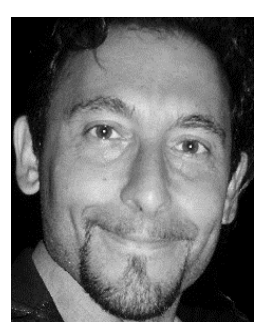

Gaetano Zizzo is Assistant Professor of Electrical Power Systems at the University of Palermo where he obtained his M.S. degree in 2002 and his Ph.D. degree in 2006. Since 2006 he works at the Department of Energy, Information Engineering and Mathematical Models of the same university. In 2017 he was elected Senior Member IEEE as a member of the Industry Application Society. He is involved in the activities of the IEEE Standard Association. His current research interests include electrical safety, power systems design, management, and optimization, smart grids and stability analysis. 